\title{
2021 EULAR recommendations regarding lifestyle behaviours and work participation to prevent progression of rheumatic and musculoskeletal diseases
}

\author{
James M Gwinnutt @ (1, ${ }^{1}$ Maud Wieczorek, ${ }^{2}$ Andra Balanescu, ${ }^{3}$ \\ Heike A Bischoff-Ferrari, ${ }^{4,5,6}$ Annelies Boonen ำ ${ }^{7,8}$ Giulio Cavalli (1) ${ }^{9}$ \\ Savia de Souza (10, ${ }^{10}$ Annette de Thurah (1) , 11,12 Thomas E Dorner, ${ }^{13,14,15}$ \\ Rikke Helene Moe (1) ${ }^{16}$ Polina Putrik (1) ${ }^{7,8}$ Javier Rodríguez-Carrio, ${ }^{17,18}$ \\ Lucía Silva-Fernández, ${ }^{19}$ Tanja Stamm (10, ${ }^{20,21}$ Karen Walker-Bone, ${ }^{22}$ Joep Welling ${ }^{23}$ \\ Mirjana I Zlatković-Švenda, ${ }^{24,25}$ Francis Guillemin, ${ }^{2,26}$ Suzanne M M Verstappen 1,22,27
}

\section{Handling editor Josef $S$ Smolen}

For numbered affiliations see end of article.

\section{Correspondence to}

Dr Suzanne M M Verstappen, Centre for Epidemiology Versus Arthritis, Faculty of Biology, Medicine and Health, The University of Manchester, Manchester, M13 9PT, UK; Suzanne.Verstappen@ manchester.ac.uk

Received 17 December 2021 Accepted 4 February 2022

\section{Check for updates}

(C) Author(s) (or their employer(s)) 2022. No commercial re-use. See rights and permissions. Published by BMJ.

To cite: Gwinnutt JM, Wieczorek M,

Balanescu A, et al.

Ann Rheum Dis Epub ahead of print: [please include Day Month Year]. doi:10.1136/ annrheumdis-2021-222020

\section{ABSTRACT}

Objectives A European League Against Rheumatism taskforce was convened to review the literature and develop recommendations on lifestyle behaviours for rheumatic and musculoskeletal diseases (RMDs). Methods Six lifestyle exposures (exercise, diet, weight, alcohol, smoking, work participation) and seven RMDs (osteoarthritis, rheumatoid arthritis, axial spondyloarthritis, psoriatic arthritis, systemic lupus erythematosus, systemic sclerosis, gout) were considered. The taskforce included health professionals in rheumatology, geriatricians, epidemiologists, public health experts, people with RMDs and exposure domain experts. Systematic reviews were conducted to gather available evidence, from which recommendations were developed.

Results Five overarching principles and 18 specific recommendations were defined based on available evidence. The overarching principles define the importance of a healthy lifestyle, how lifestyle modifications should be implemented, and their role in relation to medical treatments. Exercise recommendations highlight the safety and benefits of exercise on pain and disability, particularly among people with osteoarthritis and axial spondyloarthritis. The diet recommendations emphasise the importance of a healthy, balanced diet for people with RMDs. People with RMDs and health professionals should work together to achieve and maintain a healthy weight. Small amounts of alcohol are unlikely to negatively affect the outcomes of people with RMDs, although people with rheumatoid arthritis and gout may be at risk of flares after moderate alcohol consumption. Smokers should be supported to quit. Work participation may have benefits on RMD outcomes and should be discussed in consultations. Conclusions These recommendations cover a range of lifestyle behaviours and can guide shared decision making between people with RMDs and health professionals when developing and monitoring treatment plans.

Rheumatic and musculoskeletal diseases (RMDs) comprise a significant proportion of the burden of disability in Europe, ${ }^{12}$ which in turn negatively affects the quality of life of those people with RMDs. While some RMDs have efficacious pharmacological treatments that reduce disease activity and hence improve disability (eg, rheumatoid arthritis (RA), ${ }^{3}$ gout $^{4}$ ) others do not (eg, osteoarthritis $\left.(\mathrm{OA})^{5}\right)$. In addition to pharmacological interventions, there are increasing requests from patients, health professionals and policy-makers for more information on how changes in lifestyle alongside more effective disease management may prevent progression of RMDs and associated disability and comorbidities.

The World Health Organisation (WHO) states that all adults should aim for a healthy, balanced diet containing fruits, vegetables, nuts and whole grains, and limited free sugar, fat and salt ${ }^{6}$; that physical activity is fundamental to health and physical inactivity is a major global health risk $^{7}$; that obesity leads to negative health consequences but can be prevented ${ }^{8}$; that tobacco users need to quit ${ }^{9}$ and that harmful alcohol consumption is a cause of more than 200 disease and injury conditions. ${ }^{10}$ These are clear messages for the general population, but for people with RMDs these aspects of health have not been prioritised in strategies to promote musculoskeletal health. This is in part because available evidence has not been sufficiently collated and synthesised, and in part because there has not been a coherent public health strategy for promoting musculoskeletal health in primary, secondary or tertiary healthcare sectors. Furthermore, there is heterogeneity in information materials on lifestyle factors across countries and often these materials are not evidence based, are very generic, and may be limited to certain lifestyle domains. Individual socioeconomic factors (including health literacy) may further determine the access to and understanding of information people with RMDs receive, possibly limiting changes in lifestyle behaviour. 
While there have recently been recommendations regarding some of these lifestyle factors in RMDs, such as the 2018 European League Against Rheumatism (EULAR) recommendations for physical activity in inflammatory arthritis and OA, ${ }^{11}$ in general there is a lack of consensus regarding which lifestyle modifications can meaningfully modify RMD-specific progression (including both modifiable (eg, pain, fatigue) and irreversible (eg, joint damage) outcomes). Nevertheless, people with RMDs frequently ask about this topic, and thus it is a priority. Therefore, a EULAR taskforce (TF) was convened to synthesise the available evidence regarding lifestyle and appropriate behaviour modification in seven common inflammatory and noninflammatory RMDs with a high socioeconomic burden (OA, $\mathrm{RA}$, axial spondyloarthritis (axSpA), psoriatic arthritis (PsA), systemic lupus erythematosus (SLE), systemic sclerosis (SSc) and gout) and produce evidence-based recommendations on what lifestyle behaviour modifications can contribute to improving disease-specific outcomes such as disease activity and disability.

\section{METHODS}

These recommendations were developed following EULAR's standard operating procedure. ${ }^{12}$ A multidisciplinary TF $(\mathrm{N}=19)$ was convened which included health professionals in rheumatology, geriatricians, epidemiologists, public health experts, people with RMDs, two representatives from the Emerging EULAR Network (EMEUNET), and exposure domain experts (eg, experts on nutrition, exercise, work participation) from 11 different European countries. A steering group managed the TF (convenor: SMMV; methodologist: FG; fellows: JMG and MW).

During the first teleconference with the TF in June 2018, the protocol was discussed and the search strategies for the systematic reviews were defined to assess the association between the included exposures (diet, exercise, weight loss, smoking and working) and progression of the RMDs of interest (OA, RA, axSpA, PsA, SLE and SSc). During this meeting, the TF decided to also include gout as one of the RMDs, due to the high prevalence and alleged relation to lifestyle.

A systematic review was then conducted by JMG, MW, JRC and GC, aiming to identify systematic reviews and meta-analyses on these RMDs and exposures published between 1 January 2013 and 18 September 2018. A report of this review was written and presented to the TF at a teleconference in January 2019. At this teleconference, it was decided that alcohol should also be included as an exposure. Furthermore, since there were numerous recently-published systematic reviews and metaanalyses on $\mathrm{OA}$ and exercise as well as certain dietary exposures, it was decided that a systematic review of original articles did not need to be performed for these exposures in patients with OA. ${ }^{13-17}$

From March 2019 to May 2019, systematic reviews of original research were conducted, one for each of the six predefined exposures (excluding OA for certain exposures, as mentioned above). ${ }^{18-21}$ Population, Intervention, Comparison, Outcome (PICO) strategies were written and search strategies were developed for each review. These strategies were implemented in the PubMed/Medline, EMBASE and CENTRAL databases. Inclusion criteria were: (1) patients with a confirmed RMD of interest, (2) the study assessed the association between an included exposure and disease progression, and (3) the study was longitudinal (either experimental or observational). Each review was conducted by two members of the TF (diet: JMG and JRC; exercise: JMG and GC; weight loss: JMG and SMMV; Smoking: MW and Maxime Ransay (public health resident at the University of Lorraine); Alcohol: MW and JMG; Work: MW and PP), with oversight from the methodologist (FG). First, titles and abstracts were screened in pairs, with adjudication of any disagreements by a third member of the TF. Subsequently, full manuscripts of abstracts fulfilling the inclusion criteria were then assessed by the lead reviewer, with the second reviewer in each pair applying the inclusion criteria to the full texts of $10 \%$ of the included abstracts to assess agreement on the inclusion of full-texts, with good agreement demonstrated. Data were extracted from full manuscripts that met the inclusion and exclusion criteria by the lead reviewer. The quality of each study was assessed using the abbreviated version of the Cochrane Risk of Bias tool ${ }^{22}$ for randomised controlled trials (RCTs) (assessing random sequence generation, allocation concealment, blinding of participants, blinding of assessors) and the Quality in Prognostic Studies ${ }^{23}$ tool for observational studies. The application of the Cochrane Risk of Bias tool was aided by a machine learning algorithm that identifies passages and estimates a grade for each of the categories. This has been demonstrated to speed up the quality assessment process. ${ }^{24}$ A reviewer checked each of the algorithm's estimates and the passages that the algorithm used to make these estimates, and if there was disagreement the reviewer changed the grade.

In May 2019, a two-day face-to-face meeting was held in which the results of the systematic reviews were presented to the TF. Due to the high volume of information found, results from four out of six exposures were evaluated at this meeting (ie, diet, work participation, exercise and weight) and preliminary overarching points and recommendations were drafted. The results of the systematic reviews about the remaining two exposures (ie, smoking and alcohol) were discussed during a teleconference with the entire TF in September 2019. From May 2019 to September 2019, the results of the systematic reviews were written up into reports and distributed to the TF. In October 2019, a second face-to-face meeting was held, in which the final overarching points and recommendations were written and agreed on by all attending. Those who were unable to attend $(\mathrm{N}=7)$ were consulted beforehand to provide feedback on a draft of the recommendations.

In May 2020, a survey containing the overarching points and recommendations, as well as estimates of the strength of evidence supporting each statement (Oxford Centre for Evidence-based Medicine Levels of Evidence), ${ }^{12}$ was sent by email to all TF members asking them to rate their level of agreement (LoA) for each overarching principle (OP)/recommendation on a scale from 0 (totally disagree) to 10 (totally agree). Ideas for future research were also elicited from TF members through this survey.

\section{RESULTS}

Six systematic reviews were performed for each individual exposure to gather evidence to develop the recommendations. The studies were a combination of RCTs, nonrandomised trials and longitudinal observational studies. There was a large variation in the quality of the studies analysed. Many studies of exercise were rated as at low risk of bias, whereas many of the studies on diet were rated as having a high risk of bias (including potential conflicts of interest of study sponsors and incomplete descriptions of the randomisation and blinding processes).

The TF agreed on 5 OPs and 18 specific recommendations regarding the individual lifestyle factors (exercise $=7$; 
Table 1 Overarching principles and exposure-specific recommendations regarding lifestyle factors for people with RMDs

\begin{tabular}{lll}
$\begin{array}{l}\text { Level of } \\
\text { evidence }^{*}\end{array}$ & $\begin{array}{l}\text { Grade of } \\
\text { recommendation* }\end{array}$ & $\begin{array}{l}\text { Level of agreement, mean (SD) } \\
(\% \text { who rated } \geq 7)\end{array}$ \\
\hline 5 & D & $9.4(1.2)(94.7)$ \\
5 & D & $9.9(0.5)(100)$ \\
5 & D & $9.3(1.3)(94.7)$ \\
5 & D & $9.5(1.1)(94.7)$ \\
5 & D & $9.7(0.7)(100)$
\end{tabular}

lifestyle factors

\section{Exercise}

1. Exercise is beneficial for many health outcomes, including but not limited to RMD symptoms and progression

2. People with RMDs should exercise because of the benefits on pain, function and quality of life

$\begin{array}{lll}\text { 1a } & \text { A } & 9.9(0.3)(100) \\ \text { 1a } & \text { A } & 9.7(0.7)(100) \\ 5 & \text { D } & 9.9(0.5)(100) \\ \text { 1a } & \text { A } & 9.5(0.8)(100) \\ \text { 1a } & \text { A } & 9.9(0.3)(100) \\ \text { 1a } & \text { A } & 9.3(1.4)(94.7)\end{array}$

3. People with RMDs should avoid physical inactivity; they should engage in regular exercise according to their abilities

4. People with RMDs should perform both aerobic and strengthening exercises aiming for at least moderate intensity

5. People with RMDs should be advised that exercise is safe and that it is never too late to start exercising

6. Exercise can be performed in different settings, alone or in groups. There is a slight benefit favouring group exercises over exercises performed alone

7. People with osteoarthritis and axial spondyloarthritis should be especially encouraged to exercise as it 1a is particularly beneficial for disease related outcomes

A $\quad 9.5(0.8)(100)$

Diet

1. A healthy, balanced diet is integral to lifestyle improvement for people with RMDs

2. People with RMDs should be informed that consuming specific food types is unlikely to have large benefits for RMD outcomes

Weight

1. People with RMDs should aim for a healthy weight

2. People with RMDs who are overweight or obese should work with health professionals to achieve controlled and intentional weight loss through healthy diet and increased physical activity as this may be beneficial for RMD outcomes

\section{Alcohol}

1. The alcohol consumption of people with RMDs should be discussed together with health professionals, particularly when starting new treatments

2. People with RMDs can be reassured that a low level of alcohol consumption is unlikely to negatively impact RMD outcomes except in certain situations

3. People with rheumatoid arthritis and health professionals should be aware that moderate alcohol consumption is associated with increased risk of rheumatoid arthritis flare and comorbidities

4. People with gout and health professionals should be aware that moderate alcohol consumption is associated with increased risk of gout flare

\section{Smoking}

1. People with RMDs should be encouraged to stop smoking and be informed that smoking is detrimental to symptoms, function, disease activity, disease progression and occurrence of comorbidities in all RMDs

2. People with rheumatoid arthritis and health professionals should be aware that smoking may affect $\quad 2 a$ disease modifying anti-rheumatic drug treatment response

Work

1. Work participation may have beneficial effects on health outcomes of people with RMDs and therefore should receive attention within healthcare consultations

$\begin{array}{lll}5 & \text { D } & 9.5(1.0)(100) \\ 1 a & \text { A } & 9.7(0.8)(100) \\ & & \\ 5 & D & 9.7(0.7)(100) \\ 2 a & B & 9.5(1.6)(94.7)\end{array}$

*Level of evidence defined by the EULAR SOP: 1a: systematic review with homogeneity of RCTs, 2a: Systematic review with homogeneity of cohort studies, 5: expert opinion; Grade of recommendation: A: consistent level 1 studies, B: consistent level 2 or 3 studies or extrapolations from level 1 studies, D: level 5 evidence or troublingly inconsistent or inconclusive studies of any level.

EULAR, European League Against Rheumatism; RCTs, randomised controlled trials; RMD, rheumatic and musculoskeletal disease; SD, standard deviation; SOP, standard operating procedure.

diet $=2$; weight $=2$; alcohol $=4$; smoking $=2$; working $=1$ ) (table 1). All 19 members of the TF rated their LoA for each $\mathrm{OP} /$ recommendation. The mean LoA was $>9$ for all recommendations indicating high agreement other than one alcohol recommendation, which had a mean LoA of 8.0 (SD: 2.2).

\section{Overarching principles}

OP 1: Lifestyle improvements complement medical treatment and do not replace it

For the RMDs assessed, there are efficacious pharmacological and non-pharmacological (eg, physiotherapy) treatments available. $^{25-28}$ The evidence underpinning the following 
recommendations are studies that assessed lifestyle modifications alongside medical treatment. Therefore, all lifestyle modifications should be seen as complementary to these medical treatments and not as replacements.

\section{OP 2: Lifestyle improvements are an essential part of RMD} management and add to overall health benefits

Even where there are few data regarding the effect of lifestyle modifications on RMD-specific outcomes or where the data suggest the effect of lifestyle modifications may be small, making positive lifestyle changes for people with RMDs is important for overall health and well-being. ${ }^{29-31}$ Therefore, assessment and encouragement to make positive lifestyle modifications should be an important part of RMD management and education. The benefit of lifestyle modifications may be enhanced if people with RMDs can make positive changes in more than one aspect of their lifestyle.

\section{OP 3: World Health Organisation recommendations for a healthy} lifestyle are also applicable to people with RMDs

The WHO has provided many useful resources and recommendation reports regarding lifestyle factors for all people (available online), ${ }^{6-10}$ and these resources and recommendations are relevant and useful starting points for individuals with RMDs.

OP 4: Lifestyle recommendations for each individual with an RMD depend on factors such as age, sex, health condition, pregnancy and comorbidities

The majority of research studies included in the systematic reviews provide overall estimates of the efficacy of lifestyle modifications, without analysing subgroup variation (often justifiably given the limited power of some studies). Furthermore, some studies excluded whole subgroups of the RMD population (eg, pregnant women, people over a certain age, people with severe RMDs or comorbidities). These and other factors are likely to influence the effectiveness of lifestyle modification and therefore health professionals need to consider these factors when providing education about changes in lifestyle behaviours.

\section{OP 5: There should be regular discussions between people with RMDs and health professionals regarding lifestyle factors} Lifestyle modifications potentially have substantial benefits to overall and RMD-specific health and should be a recurrent and frequent theme in discussions between people with RMDs and health professionals. Furthermore, given the complexity of RMD conditions (eg, early vs established disease, disease flares) and the potential interactions between lifestyle modifications, current health condition and medical treatment, people with RMDs may need guidance and support from health professionals to implement a combination of healthy lifestyle changes. ${ }^{32-34}$ This guidance and support should stem from a comprehensive management plan known to all health professionals directly involved in the disease management of people with RMDs.

\section{Exposure-specific recommendations}

Exercise 1: Exercise is beneficial for many health outcomes, including but not limited to RMD symptoms and progression

As stated previously, exercise is beneficial for a multitude of general health outcomes and the WHO and US Department of Health and Human Services have provided general guidelines regarding exercise. ${ }^{735}$ These benefits have also been shown for RMD-specific outcomes, such as pain and disability. Health professionals should collaborate with people with RMDs to make shared decisions regarding the type of activity that is appropriate for their ability and condition. EULAR has previously published recommendations for physical activity for people with OA and inflammatory arthritis, and our TF endorses these recommendations. ${ }^{11}$

Exercise 2: People with RMDs should exercise because of the benefits on pain, function and quality of life

There have been many research studies reporting the benefits of exercise on RMD-specific outcomes, with the general consensus being that exercise is beneficial for pain, function, fatigue and health-related quality of life in RMDs. ${ }^{14} 17$ 36-41 The evidence is stronger for short-term (6-12 months) benefits compared with long-term benefits, although long-term benefits have been demonstrated for the general population on a range of health outcomes and thereby can be extrapolated to people with RMDs. ${ }^{42}$ Exercise may be effective at modifying disease activity in some conditions (eg, axSpA), although to a smaller extent. $^{404344}$ Furthermore, research has primarily focused on OA, RA and axSpA, with far fewer research studies on SLE, PsA, SSc and gout.

Exercise 3: People with RMDs should avoid physical inactivity; they should engage in regular exercise according to their abilities Sedentary behaviour is associated with many negative health outcomes in the general population. ${ }^{45-47}$ These negative outcomes will also affect people with RMDs. Therefore, health professionals should encourage people with RMDs to avoid a lifestyle characterised by prolonged periods of physical inactivity.

Exercise 4: People with RMDs should perform both aerobic and strengthening exercises aiming for at least moderate intensity In line with previous recommendations, ${ }^{1135}$ evidence suggests that both aerobic and strengthening exercises are beneficial for people with RMDs, and therefore, both should be included when exercising. ${ }^{14174048-50}$ People with RMDs should aim for at least moderate intensity aerobic exercise $(64 \%-76 \%$ of maximal heart rate $^{51}$ ) for at least $150 \mathrm{~min}$ per week, and strengthening exercise $(50 \%-69 \%$ of an individual's one-repetition maximal effort) twice a week. ${ }^{35}$

Exercise 5: People with RMDs should be advised that exercise is safe and that it is never too late to start exercising

Among studies that reported about adverse events from exercise interventions in RMDs, many reported no or very few adverse events related to the interventions. ${ }^{52-56}$ People with RMDs should be reminded that exercise is a safe way to improve their health condition, and that it is never too late to take up exercise, even if people had been less physically active prior to the onset of their condition.

Exercise 6: Exercise can be performed in different settings, alone or in groups. There is a slight benefit favouring group exercises over exercises performed alone

People with RMDs should be encouraged to perform the exercise that suits them best, whether that be alone at home or in exercise classes. There is some evidence that supervised exercise providing tutoring and peer-support may be marginally more effective than individual exercise, ${ }^{57-60}$ but this should not discourage individuals from performing home-based exercise if they are unable to travel or unwilling to attend classes, as performing exercise that interests individuals and fits into their schedules is likely to lead to increased adherence. 
Exercise 7: People with osteoarthritis and axial spondyloarthritis should be especially encouraged to exercise as it is particularly beneficial for disease related outcomes

While exercise is beneficial for all RMDs, people with OA and axSpA should be particularly encouraged to exercise, as the results of studies of exercise in these conditions appear to show strong and consistent effects on reducing pain and improving function. ${ }^{141617406162}$

Diet 1: A healthy, balanced diet is integral to lifestyle improvement for people with RMDs

It is important that patients with RMDs are educated about the benefits of maintaining a healthy, balanced diet. Imbalances in energy intake and expenditure contribute to obesity increasing the risk of chronic diseases (eg, cardiovascular disease, type 2 diabetes mellitus) in the general population and also in people with RMDs. Quality of food is also linked to nutrition-related diseases. The WHO recommend that people should eat less high-calorie food, especially high in saturated or trans fats and sugars; eat more fruits, vegetables and legumes, and select foods of plant and marine origin. ${ }^{6}$

\section{Diet 2: People with RMDs should be informed that consuming} specific food types is unlikely to have large benefits for RMD outcomes

There have been many studies investigating the clinical efficacy of administering specific food types or components (eg, nutrients) in people with RMDs. Mostly, these studies have been small scale or pilot studies, resulting in imprecise estimates and therefore reliable conclusions cannot be drawn. For some food components, larger studies have been conducted (eg, vitamin $\mathrm{D}$ for $\mathrm{OA}^{63-65}$; marine oil/omega 3 for $\mathrm{RA}^{66}{ }^{67}$ ) which have reported small effect sizes that are unlikely to be clinically meaningful. While a high purine diet is a risk factor for the onset of gout, there is little clinical trial data on the efficacy of lifestyle modifications in people with gout. ${ }^{4}$ However, the 2016 EULAR guidelines on the management of gout recommend people with gout to avoid sugar-sweetened drinks, heavy meals and excessive intake of meat and seafood as well as encouraging low-fat dairy products $^{68}$-this TF supports these recommendations. Overall, it is unlikely that consumption of specific food types will have large effects on RMD-specific outcomes.

\section{Weight 1: People with RMDs should aim for a healthy weight}

As part of a healthy lifestyle, people with RMDs should aim for a healthy weight. Communication of what constitutes a healthy weight for each individual should be based on shared decision-making within a multidisciplinary team and should take into account age, sex, disease state, treatment and comorbidity. When people gain or lose weight, adjustments in medication (eg, dosage, frequency) or clinical status (eg, comorbidity occurrence, flare) should be considered.

Weight 2: People with RMDs who are overweight or obese should work with health professionals to achieve controlled and intentional weight loss through healthy diet and increased physical activity as this may be beneficial for RMD outcomes

Being overweight/obese is associated with worse RMD specific outcomes, ${ }^{69-73}$ and weight loss can improve RMD specific outcomes $^{74-76}$ as well as other health-related outcomes. ${ }^{77-79}$ In adults, a body mass index of $\geq 25 \mathrm{~kg} / \mathrm{m}^{2}$ is considered overweight and $\geq 30 \mathrm{~kg} / \mathrm{m}^{2}$ is considered obese by the WHO. ${ }^{80}$
Alcohol 1: The alcohol consumption of people with RMDs should be discussed together with health professionals, particularly when starting new treatments

Discussions with people with RMDs about their alcohol consumption should be initiated by health professionals, particularly when starting specific new treatments such as methotrexate. People with RMDs should be aware of the negative health consequences of overconsumption of alcohol, ${ }^{81} 82$ and steps should be taken to curtail unhealthy alcohol usage.

Alcohol 2: People with RMDs can be reassured that a low level of alcohol consumption is unlikely to negatively impact RMD outcomes except in certain situations

While there have been relatively few research studies on alcohol and RMDs, those that have been published have shown that, in general, low levels of alcohol consumption are unlikely to have negative impacts on RMD-specific outcomes, ${ }^{83-85}$ except in certain situations such as those with liver disease or who use certain treatments (eg, methotrexate, leflunomide).

Alcohol 3: People with rheumatoid arthritis and health professionals should be aware that moderate alcohol consumption is associated with increased risk of rheumatoid arthritis flare and comorbidities Several research studies have indicated that moderate alcohol consumption may increase the risk of comorbidities and increase disease activity in people with RA. ${ }^{86-88}$ Health professionals should discuss the risks of alcohol consumption with people with RA.

Alcohol 4: People with gout and health professionals should be aware that moderate alcohol consumption is associated with increased risk of gout flare

Several research studies have indicated that alcohol consumption is associated with an increased risk of gout attacks. ${ }^{89}$ Health professionals should discuss the risks of alcohol consumption with people with gout.

Smoking 1: People with RMDs should be encouraged to stop smoking and be informed that smoking is detrimental to symptoms, function, disease activity, disease progression and occurrence of comorbidities in all RMDs

Smoking is a well-established risk factor for many negative health outcomes. ${ }^{90-92}$ Research studies of people with RMDs also indicate that smoking has a negative impact on many of their health outcomes, such as disease activity, function, progression and occurrence of comorbidities. ${ }^{93-97}$ People with RMDs who smoke should be made aware that continuing to smoke is likely to lead to worse symptom burden as well as increased risk of serious comorbidity, such as cardiovascular disease as outlined by a 2016 EULAR TF on cardiovascular disease risk management. ${ }^{98}$ These people should be offered support to quit and progress towards quitting should be monitored by health professionals.

Smoking 2: People with rheumatoid arthritis and health professionals should be aware that smoking may affect disease modifying anti-rheumatic drug treatment response

There is some evidence that smoking may limit response to DMARDs in RA. ${ }^{99-101}$ Therefore, health professionals should make people with RA who smoke aware of this effect and support smokers towards quitting. 


\begin{tabular}{ll}
\hline Table 2 & Research agenda \\
\hline Item & Research area \\
\hline $1 . \quad \begin{array}{l}\text { To address the large imbalance in the available evidence across the diseases } \\
\text { within the scope of these recommendations, more high quality studies are } \\
\text { needed on SLE, PsA, SSc and gout for all the lifestyle exposures included } \\
\text { in this TF. Larger, high-quality studies are needed for all RMDs on certain } \\
\text { lifestyle exposures (such as diet, where effect sizes are likely to be small). }\end{array}$ \\
$\begin{array}{ll}\text { To understand the synergistic effect of multiple lifestyle improvements on } \\
\text { disease progression. }\end{array}$ \\
$\begin{array}{l}\text { To gain a greater understanding of the effect of socioeconomic deprivation } \\
\text { on the associations between lifestyle factors and the outcomes assessed in } \\
\text { this TF. }\end{array}$ \\
\hline
\end{tabular}

PsA, psoriatic arthritis; RMDs, rheumatic and musculoskeletal diseases; SLE, systemic lupus erythematosus; SSc, systemic sclerosis; TF, taskforce.

Work 1: Work participation may have beneficial effects on health outcomes of people with RMDs and therefore should receive attention within healthcare consultations

There have been several studies about work participation in people with RMDs, including studies of interventions aiming to keep people in work. ${ }^{102}$ While there are some inconsistencies between these studies, perhaps due to power and different outcome assessments, many indicated that work was probably not detrimental (although there is evidence that physically demanding jobs are associated with increased radiographic progression in $\operatorname{axSpA}^{103}{ }^{104}$ ) and in some cases beneficial for people with RMDs in terms of RMD outcomes. ${ }^{105-107}$ Given this, and the benefits of work on quality of life seen in the general population, ${ }^{108}$ work should receive increased attention in healthcare consultations with people with RMDs, and specialised health professionals should be involved. Moreover, institutions in the health and social sector, employers and patient organisations should offer support regarding work participation.

\section{Research agenda}

The amount of research performed regarding lifestyle exposures and outcomes varied between the RMDs covered by this TF. The majority of the research had been performed in RA and OA, and to a lesser extent in axSpA. However, there is limited evidence on lifestyle exposures and progression of disease outcomes for populations with SLE, PsA, SSc and gout. Good quality research is needed for all the lifestyle exposures included in this TF for SLE, PsA, SSc and gout (table 2). Furthermore, many of the studies investigating diet were of low quality, being nonrandomised trials or very-small scale pilot studies. Given that the effect of singular dietary exposures is likely to be small, large well-designed trials with long follow-up are necessary.

A vast majority of studies included in the reviews of this TF investigated each lifestyle exposure individually. More research is needed to understand the synergistic effect of multiple lifestyle interventions given how intrinsically linked many of these lifestyle factors are, such as diet, exercise and weight (table 2).

The accumulation of multiple unhealthy lifestyle behaviours is also likely deleterious, and this accumulation may be influenced by people's personal and environmental context. For instance, people with lower socioeconomic status are more likely to eat poorly, ${ }^{109} 110$ exercise less, ${ }^{111} 112$ be obese, ${ }^{113}{ }^{114}$ smoke more ${ }^{115}$ and experience more adverse outcomes from alcohol. ${ }^{116}$ They are also more likely to be employed in physically demanding jobs. ${ }^{117}$ There are also disease-specific barriers to making lifestyle changes. ${ }^{118}$ The influence these have on the association between lifestyle and RMD progression is not clear and a greater understanding of this may lead to improved targeting of lifestyle advice and interventions (table 2).

\section{Evaluation and dissemination}

We will aim to evaluate these recommendations by conducting an online survey across several European countries, collecting data on the feasibility, acceptability and transparency of the recommendations. To disseminate these recommendations, we will develop information materials about healthy living and the impact of lifestyle on RMD progression in collaboration with patients and patient organisations. Summaries of evidence will also be written and distributed to health professionals and stakeholder organisations (WHO, EULAR, National Institute for Health and Care Excellence).

\section{DISCUSSION}

This TF agreed on five OPs and 18 specific recommendations covering a range of important lifestyle factors and work participation for seven common RMDs. These recommendations are consistent with and build on previous WHO, US government and EULAR recommendations. ${ }^{67113580}$ These recommendations represent the consensus views of a wide spectrum of health professionals, scientists and people with RMDs and, due to the wide geographic representation of the TF members, should be appropriate for all individuals with RMDs across Europe. These recommendations are based on a broad review of the literature and thus, for the most part, are evidence-based. The level of agreement was high across the recommendations, indicating the broad acceptability of this recommendation set. While the evidence-base was primarily on individual lifestyle factors only, the TF acknowledge the potential benefit of several lifestyle changes in combination for people with RMDs.

Defining recommendations based on evidence is the first step towards improving the lifestyles, and ultimately the disease outcomes, of people with RMDs. The next step is to disseminate these recommendations as widely as possible across Europe. This will be done in the form of manuscripts, presentations, and bespoke leaflets translated into languages from across the continent.

Once these recommendations have been disseminated, people with RMDs themselves will be encouraged to implement these recommendations, with support from health professionals and/ or family and friends. First, patients with RMDs may require education on the benefits of making lifestyle behaviour changes. Then theory-driven behaviour change interventions are required to aid people with RMDs to implement these recommendations. ${ }^{119}$ System level changes are also needed - the ability to make lifestyle changes should be affordable and available to all.

Limitations of these recommendations include the breath of the scope of the project; including six exposures across seven RMDs necessitated a series of large systematic reviews. Consequently, findings from more than 700 papers were included. While this surely represents a significant proportion of the research on these exposures, there is the possibility that some studies were missed. Furthermore, there is likely some publication bias in the selected papers, particularly for certain exposures. For many dietary exposures, only one small-scale study with a large effect size was found, indicating potential selective reporting. Furthermore, for some dietary exposures, there were concerns regarding conflicts of interest, with overlap between companies producing the dietary exposures and sponsoring the studies. For some exposures, the main evidence base was derived from observational studies, given that it would be unethical to 
test these exposures within interventional studies (eg, smoking, alcohol). Appropriate caution was used when assessing these observational studies (due to the difficulties inferring causation from observational data) while devising the recommendations. Lastly, there was a large disparity in the amount of evidence published between the included RMDs. While we have tried to be general in the majority of the recommendations, and believe that each recommendation is relevant for all the included RMDs, necessarily some recommendations will be supported by greater or lesser amounts of evidence depending on the RMD.

The research agenda highlighted the lack of high-quality, homogeneous studies for certain exposures (eg, diet) and the imbalance in the amount of studies across the included RMDs. Also, greater understanding is needed regarding how various lifestyle factors interact to produce positive or negative outcomes in RMDs, as research to date has primarily focused on each lifestyle exposure in isolation. The delivery of future lifestyle interventions as packages aiming to influence all aspects of lifestyle should be evaluated, given the inter-relatedness of these exposures. Lastly, the influence of personal and environmental context, such as socioeconomic deprivation, on the associations between lifestyle exposures, work participation and outcomes requires more research, given the potentially large influence this has on exposure to negative lifestyle factors.

In conclusion, we have developed an extensive set of recommendations that can serve as a foundation for discussion and shared decision-making regarding positive modifications to the lifestyles of people with RMDs, with the ultimate goal being improvements in symptoms, quality of life and long-term outcomes.

\section{Author affiliations}

Centre for Epidemiology Versus Arthritis, Faculty of Biology, Medicine and Health, The University of Manchester, Manchester, UK

${ }^{2} E A 4360$ Apemac, Université de Lorraine, Nancy, France

${ }^{3}$ Department of Internal Medicine and Rheumatology, "Sf. Maria" Hospital, "Carol Davila" University of Medicine and Pharmacy, Bucharest, Romania

${ }^{4}$ Department of Aging Medicine and Aging Research, University Hospital Zurich and University of Zurich, Zurich, Switzerland

${ }^{5}$ Center on Aging and Mobility, University of Zurich, Zurich, Switzerland

${ }^{6}$ University Clinic for Aging Medicine, City Hospital Zurich - Waid, Zurich, Switzerland

${ }^{7}$ Care and Public Health Research Institute (CAPHRI), Maastricht University, Maastricht, The Netherlands

${ }^{8}$ Department of Internal Medicine, Division of Rheumatology, Maastricht University Medical Center, Maastricht, The Netherlands

${ }^{9}$ Unit of Immunology, Rheumatology, Allergy and Rare Diseases, IRCCS San Raffaele Hospital and Vita-Salute San Raffaele University, Milan, Italy

${ }^{10}$ Centre for Rheumatic Diseases, King's College London, London, UK

${ }^{11}$ Department of Clinical Medicine, Aarhus University, Aarhus, Denmark

${ }^{12}$ Department of Rheumatology, Aarhus University Hospital, Aarhus, Denmark

${ }^{13}$ Centre for Public Health, Department of Social and Preventive Medicine, Medical University of Vienna, Vienna, Austria

${ }^{14}$ Social Insurance Fund for Public Service, Railway and Mining Industries, SitzenbergReidling, Austria

${ }^{15}$ Karl-Landsteiner Institute for Health Promotion Research, Sitzenberg-Reidling, Austria

${ }^{16}$ National Advisory Unit for Rehabilitation in Rheumatology, Division of Rheumatology and Research, Diakonhjemmet Hospital, Oslo, Norway

${ }^{17}$ Area of Immunology, Department of Functional Biology, Universidad de Oviedo, Oviedo, Spain

${ }^{18}$ Department of Metabolism, Instituto de Investigación Sanitaria del Principado de Asturias (ISPA), Oviedo, Spain

${ }^{19}$ Rheumatology Department, Hospital Universitari Son Espases, Palma de Mallorca, Spain

${ }^{20}$ Section for Outcomes Research, Center for Medical Statistics, Informatics, and Intelligent Systems, Medical University of Vienna, Vienna, Austria

${ }^{21}$ Ludwig Boltzmann Institute for Arthritis and Rehabilitation, Vienna, Austria

${ }^{22}$ MRC Versus Arthritis Centre for Musculoskeletal Health and Work, University of

Southampton, Southampton, UK

${ }^{23}$ NVLE Dutch Patient Organization for Systemic Autoimmune Diseases, Utrecht, The Netherlands
${ }^{24}$ Institute of Rheumatology, University of Belgrade School of Medicine, Belgrade, Serbia

${ }^{25}$ Department of Internal Medicine, University of East Sarajevo Faculty of Medicine Foča, Republika Srpska, Bosnia and Herzegovina

${ }^{26}$ Inserm, CHRU Nancy, CIC-1433 Epidémiologie Clinique, Université de Lorraine, Nancy, France

${ }^{27}$ NIHR Manchester Biomedical Research Centre, Manchester University NHS Foundation Trust, Manchester Academic Health Science Centre, Manchester, UK

Twitter James M Gwinnutt @james_gwinnutt and Rikke Helene Moe @ MoeRikke

Acknowledgements The Taskforce would like to thank the library teams at the University of Lorraine and the University of Manchester, especially Mary Ingram at the Centre for Epidemiology Versus Arthritis, for their help with the literature reviews. Thanks to Maxime Ransay for help with the systematic review on smoking. Thanks also to the administrative team of the Division of Musculoskeletal and Dermatological Science at the University of Manchester for their help with organising the face-to-face meetings.

Contributors JMG, MW, FG and SMMV contributed equally. SMMV conceived the idea and submitted the project with a contribution from FG to the EULAR executive committee. JMG and MW were the research fellows on the project who carried out the systematic literature reviews, with the help of PP, Maxime Ransay and EMEUNET members GC and JRC. These reviews were overseen by FG (methodologist) and SMMV (convenor). SMMV organised and chaired the telephone and face-to-face meetings. All authors contributed to the drafting of recommendations at the telephone and face-to-face meetings and helped to revise the recommendations manuscript.

Funding SMMV and JMG are supported by Versus Arthritis (21755) and the NIHR Manchester Biomedical Research Centre. The views expressed are those of the author(s) and not necessarily those of the NHS, the NIHR or the Department of Health. JRC is supported by the Juan de la Cierva (IJCI-2017-32070) and Sara Borrell (CD19/00120) programs. The taskforce would like to thank EULAR for providing financial support for this project.

\section{Competing interests None declared.}

Patient and public involvement Patients and/or the public were involved in the design, or conduct, or reporting, or dissemination plans of this research. Refer to the Methods section for further details.

Patient consent for publication Not applicable.

Ethics approval This study does not involve human participants.

Provenance and peer review Not commissioned; externally peer reviewed.

\section{ORCID iDs}

James M Gwinnutt http://orcid.org/0000-0002-1435-8797

Annelies Boonen http://orcid org/0000-0003-0682-9533

Giulio Cavalli http://orcid.org/0000-0001-8728-3004

Savia de Souza http://orcid.org/0000-0003-4953-3257

Annette de Thurah http://orcid.org/0000-0003-0103-4328

Rikke Helene Moe http://orcid.org/0000-0001-7601-5346

Polina Putrik http://orcid.org/0000-0002-9342-1861

Tanja Stamm http://orcid.org/0000-0003-3073-7284

\section{REFERENCES}

1 March L, Smith EUR, Hoy DG, et al. Burden of disability due to musculoskeletal (MSK) disorders. Best Pract Res Clin Rheumatol 2014;28:353-66.

2 Sebbag E, Felten R, Sagez F, et al. The world-wide burden of musculoskeletal diseases: a systematic analysis of the world Health organization burden of diseases database. Ann Rheum Dis 2019;78:844-8.

3 Smolen JS, Aletaha D, Barton A, et al. Rheumatoid arthritis. Nat Rev Dis Primers 2018;4:18001.

4 Dalbeth N, Choi HK, Joosten LAB, et al. Gout. Nat Rev Dis Primers 2019;5:69

5 Martel-Pelletier J, Barr AJ, Cicuttini FM, et al. Osteoarthritis. Nat Rev Dis Primers 2016:2:16072

6 World Health Organisation. Diet, nutrition and the prevention of chronic diseases. Report of the joint WHO/FAO expert consultation. who technical report series. World health organisation, 2018.

7 World Health Organisation. Global recommendations on physical activity for health, 2010.

8 World Health Organisation. Obesity and overweight factsheet, 2018. Available: https://www.who.int/en/news-room/fact-sheets/detail/obesity-and-overweight [Accessed 28 Jan 2020].

9 World Health Organisation. Who report on the global tobacco epidemic, 2019, 2019.

10 World Health Organisation. Alcohol fact sheet, 2018. Available: https://www.who. int/news-room/fact-sheets/detail/alcohol [Accessed 28 Jan 2020]. 
11 Rausch Osthoff A-K, Niedermann K, Braun J, et al. 2018 EULAR recommendations for physical activity in people with inflammatory arthritis and osteoarthritis. Ann Rheum Dis 2018;77:1251-60.

12 van der Heijde D, Aletaha D, Carmona L, et al. 2014 update of the EULAR standardised operating procedures for EULAR-endorsed recommendations. Ann Rheum Dis 2015;74:8-13.

13 Liu X, Machado GC, Eyles JP, et al. Dietary supplements for treating osteoarthritis: a systematic review and meta-analysis. Br J Sports Med 2018;52:167-75.

14 Juhl C, Christensen R, Roos EM, et al. Impact of exercise type and dose on pain and disability in knee osteoarthritis: a systematic review and meta-regression analysis of randomized controlled trials. Arthritis Rheumatol 2014;66:622-36.

15 Hurley M, Dickson K, Hallett R, et al. Exercise interventions and patient beliefs for people with hip, knee or hip and knee osteoarthritis: a mixed methods review. Cochrane Database Syst Rev 2018;4:CD010842.

16 Corbett MS, Rice SJC, Madurasinghe V, et al. Acupuncture and other physical treatments for the relief of pain due to osteoarthritis of the knee: network metaanalysis. Osteoarthritis Cartilage 2013;21:1290-8.

17 Uthman OA, van der Windt DA, Jordan JL, et al. Exercise for lower limb osteoarthritis: systematic review incorporating trial sequential analysis and network meta-analysis. BMJ 2013;347: 55555.

18 Gwinnutt JM, Wieczorek M, Rodríguez-Carrio J, et al. Effects of diet on the outcomes of rheumatic and musculoskeletal diseases (RMDs): systematic review and metaanalyses Informing the 2021 EULAR recommendations for lifestyle improvements in people with RMDs. RMD Open 2022.

19 Gwinnutt JM, Wieczorek M, Cavalli G, et al. Effects of physical exercise and body weight on disease-specific outcomes of people with rheumatic and musculoskeletal diseases (RMDs): systematic reviews and meta-analyses Informing the 2021 EULAR recommendations for lifestyle improvements in people with RMDs. RMD Open 2022.

20 Wieczorek M, Gwinnutt JM, Ransay M, et al. Smoking, alcohol consumption and disease-specific outcomes in rheumatic and musculoskeletal diseases (RMDs): systematic reviews Informing the 2021 EULAR recommendations for lifestyle improvements in people with RMDs. RMD Open 2022.

21 Wieczorek M, Verstappen SMM, Putrik P, et al. Association of vocational interventions and work-related factors with disease-specific outcomes in individuals with rheumatic and musculoskeletal diseases (RMDs): a systematic literature review Informing the 2021 EULAR recommendations for lifestyle improvements in people with RMDs 2022

22 Higgins JPT, Altman DG, Gøtzsche PC, et al. The Cochrane collaboration's tool for assessing risk of bias in randomised trials. BMJ 2011;343:d5928.

23 Hayden JA, van der Windt DA, Cartwright JL, et al. Assessing bias in studies of prognostic factors. Ann Intern Med 2013;158:280-6.

24 Soboczenski F, Trikalinos TA, Kuiper J, et al. Machine learning to help researchers evaluate biases in clinical trials: a prospective, randomized user study. BMC Med Inform Decis Mak 2019;19:96.

25 Singh JA, Christensen R, Wells GA, et al. Biologics for rheumatoid arthritis: an overview of Cochrane reviews. Cochrane Database Syst Rev 2009;4:CD007848

26 Coates LC, Cawkwell LS, Ng NWF, et al. Real life experience confirms sustained response to long-term biologics and switching in ankylosing spondylitis. Rheumatology 2008:47:897-900.

27 Lord PAC, Farragher TM, Lunt M, et al. Predictors of response to anti-TNF therapy in ankylosing spondylitis: results from the British Society for rheumatology biologics register. Rheumatology 2010;49:563-70.

28 Walsh NE, Pearson J, Healey EL. Physiotherapy management of lower limb osteoarthritis. Br Med Bull 2017:122:151-61.

29 Akbaraly TN, Ferrie JE, Berr C, et al. Alternative healthy eating index and mortality over $18 \mathrm{Y}$ of follow-up: results from the Whitehall II cohort. Am J Clin Nutr 2011;94:247-53

30 Shivappa N, Hebert JR, Kivimaki M, et al. Alternative healthy eating index 2010, dietary inflammatory index and risk of mortality: results from the Whitehall II cohort study and meta-analysis of previous dietary inflammatory index and mortality studies. Br J Nutr 2017;118:210-21.

31 Ford ES, Bergmann MM, Boeing $\mathrm{H}$, et al. Healthy lifestyle behaviors and all-cause mortality among adults in the United States. Prev Med 2012;55:23-7.

32 Carson KV, Verbiest MEA, Crone MR, et al. Training health professionals in smoking cessation. Cochrane Database Syst Rev 2012;118.

33 Veldhuijzen van Zanten JJCS, Rouse PC, Hale ED, et al. Perceived barriers, facilitators and benefits for regular physical activity and exercise in patients with rheumatoid arthritis: a review of the literature. Sports Med 2015;45:1401-12.

34 Walker RE, Kusch J, Fink JT, et al. Facilitating factors and barriers to weight management in women: physician perspectives. J Patient Cent Res Rev 2018;5:18-27.

35 Piercy KL, Troiano RP, Ballard RM, et al. The physical activity guidelines for Americans. JAMA 2018:320:2020-8.

36 Bartholdy C, Juhl C, Christensen R, et al. The role of muscle strengthening in exercise therapy for knee osteoarthritis: a systematic review and meta-regression analysis of randomized trials. Semin Arthritis Rheum 2017;47:9-21.
37 Baillet A, Vaillant M, Guinot M, et al. Efficacy of resistance exercises in rheumatoid arthritis: meta-analysis of randomized controlled trials. Rheumatology 2012;51:519-27

38 Baillet A, Zeboulon N, Gossec L, et al. Efficacy of cardiorespiratory aerobic exercise in rheumatoid arthritis: meta-analysis of randomized controlled trials. Arthritis Care Res 2010:62:984-92.

39 Millner JR, Barron JS, Beinke KM, et al. Exercise for ankylosing spondylitis: an evidence-based consensus statement. Semin Arthritis Rheum 2016:45:411-27.

40 Pécourneau V, Degboé Y, Barnetche T, et al. Effectiveness of exercise programs in ankylosing spondylitis: a meta-analysis of randomized controlled trials. Arch Phys Med Rehabil 2018;99:383-9.

41 Cramp F, Hewlett S, Almeida C, et al. Non-Pharmacological interventions for fatigue in rheumatoid arthritis. Cochrane Database Syst Rev 2013:8:CD008322.

42 Reiner M, Niermann C, Jekauc D, et al. Long-term health benefits of physical activity-a systematic review of longitudinal studies. BMC Public Health 2013;13:813-13.

43 Liang $\mathrm{H}$, Zhang $\mathrm{H}$, Ji H, et al. Effects of home-based exercise intervention on healthrelated quality of life for patients with ankylosing spondylitis: a meta-analysis. Clin Rheumatol 2015;34:1737-44.

44 Martins NA, Furtado GE, Campos MJ, et al. Exercise and ankylosing spondylitis with new York modified criteria: a systematic review of controlled trials with metaanalysis. Acta Reumatol Port 2014;39:298-308.

45 Wilmot EG, Edwardson CL, Achana FA, et al. Sedentary time in adults and the association with diabetes, cardiovascular disease and death: systematic review and meta-analysis. Diabetologia 2012;55:2895-905.

46 Ekelund U, Tarp J, Steene-Johannessen J, et al. Dose-Response associations between accelerometry measured physical activity and sedentary time and all cause mortality: systematic review and harmonised meta-analysis. BMJ 2019;366:14570-170

47 Kerr J, Anderson C, Lippman SM. Physical activity, sedentary behaviour, diet, and cancer: an update and emerging new evidence. Lancet Oncol 2017;18:e457-71.

48 Rausch Osthoff A-K, Juhl CB, Knittle K, et al. Effects of exercise and physical activity promotion: meta-analysis Informing the 2018 EULAR recommendations for physical activity in people with rheumatoid arthritis, spondyloarthritis and hip/knee osteoarthritis. RMD Open 2018;4:e000713.

49 Rongen-van Dartel SAA, Repping-Wuts $\mathrm{H}$, Flendrie $\mathrm{M}$, et al. Effect of aerobic exercise training on fatigue in rheumatoid arthritis: a meta-analysis. Arthritis Care Res 2015:67:1054-62.

50 Williams MA, Srikesavan C, Heine PJ, et al. Exercise for rheumatoid arthritis of the hand. Cochrane Database Syst Rev 2018:7:CD003832.

51 Centers for Disease Control and Prevention. Target heart rate and estimated maximum heart rate, 2020. Available: https://www.cdc.gov/physicalactivity/basics/ measuring/heartrate.htm [Accessed 07 Jan 2021].

52 Lamb SE, Williamson EM, Heine PJ, et al. Exercises to improve function of the rheumatoid hand (Sarah): a randomised controlled trial. Lancet 2015;385:421-9.

53 Katz P, Margaretten M, Gregorich S, et al. Physical activity to reduce fatigue in rheumatoid arthritis: a randomized controlled trial. Arthritis Care Res 2018;70:1-10.

54 Baillet A, Payraud E, Niderprim V-A, et al. A dynamic exercise programme to improve patients' disability in rheumatoid arthritis: a prospective randomized controlled trial. Rheumatology 2009;48:410-5.

55 Pinto ALS, Oliveira NC, Gualano B, et al. Efficacy and safety of concurrent training in systemic sclerosis. J Strength Cond Res 2011;25:1423-8.

56 Roger-Silva D, Natour J, Moreira E, et al. A resistance exercise program improves functional capacity of patients with psoriatic arthritis: a randomized controlled trial. Clin Rheumatol 2018;37:389-95.

57 Seneca T, Hauge EM, Maribo T. Comparable effect of partly supervised and selfadministered exercise programme in early rheumatoid arthritis--a randomised controlled trial. Dan Med J 2015:62:A5127.

58 Anwer S, Alghadir A, Brismée J-M. Effect of home exercise program in patients with knee osteoarthritis: a systematic review and meta-analysis. J Geriatr Phys Ther 2016;39:38-48

59 Aydın T, Tașpınar Özgür, Sarıyıldız MA, et al. Evaluation of the effectiveness of home based or hospital based calisthenic exercises in patients with ankylosing spondylitis. J Back Musculoskelet Rehabil 2016:29:723-30.

60 Analay Y, Ozcan E, Karan A, et al. The effectiveness of intensive group exercise on patients with ankylosing spondylitis. Clin Rehabil 2003;17:631-6.

61 Sveaas SH, Bilberg A, Berg IJ, et al. High intensity exercise for 3 months reduces disease activity in axial spondyloarthritis (axSpA): a multicentre randomised trial of 100 patients. Br J Sports Med 2020;54:292-7.

62 Tanaka R, Ozawa J, Kito N, et al. Efficacy of strengthening or aerobic exercise on pain relief in people with knee osteoarthritis: a systematic review and meta-analysis of randomized controlled trials. Clin Rehabil 2013;27:1059-71.

63 Arden NK, Cro S, Sheard S, et al. The effect of vitamin D supplementation on knee osteoarthritis, the video study: a randomised controlled trial. Osteoarthritis Cartilage 2016:24:1858-66

64 Bischoff-Ferrari HA, Orav EJ, Egli A, et al. Recovery after unilateral knee replacement due to severe osteoarthritis and progression in the contralateral knee: a randomised clinical trial comparing daily 2000 IU versus 800 IU vitamin D. RMD Open 2018;4:e000678. 
65 Jin $X$, Jones $G$, Cicuttini F, et al. Effect of vitamin D supplementation on tibial cartilage volume and knee pain among patients with symptomatic knee osteoarthritis: a randomized clinical trial. JAMA 2016;315:1005-13.

66 Senftleber NK, Nielsen SM, Andersen JR, et al. Marine oil supplements for arthritis pain: a systematic review and meta-analysis of randomized trials. Nutrients 2017;9. doi:10.3390/nu9010042. [Epub ahead of print: 06 Jan 2017].

67 Gioxari A, Kaliora AC, Marantidou F, et al. Intake of $\omega$-3 polyunsaturated fatty acids in patients with rheumatoid arthritis: a systematic review and meta-analysis. Nutrition 2018;45:114-24

68 Richette P, Doherty M, Pascual E, et al. 2016 updated EULAR evidence-based recommendations for the management of gout. Ann Rheum Dis 2017;76:29-42.

69 Liu Y, Hazlewood GS, Kaplan GG, et al. Impact of obesity on remission and disease activity in rheumatoid arthritis: a systematic review and meta-analysis. Arthritis Care Res 2017;69:157-65.

70 Nikiphorou E, Norton S, Young A, et al. The association of obesity with disease activity, functional ability and quality of life in early rheumatoid arthritis: data from the early rheumatoid arthritis Study/Early rheumatoid arthritis network UK prospective cohorts. Rheumatology 2018;57:1194-202.

71 Katz P, Yazdany J, Julian L, et al. Impact of obesity on functioning among women with systemic lupus erythematosus. Arthritis Care Res 2011;63:1357-64.

72 Ottaviani S, Allanore Y, Tubach F, et al. Body mass index influences the response to infliximab in ankylosing spondylitis. Arthritis Res Ther 2012;14:R115.

73 Nguyen U-SDT, Zhang Y, Louie-Gao Q, et al. Obesity paradox in recurrent attacks of gout in observational studies: clarification and remedy. Arthritis Care Res 2017;69:561-6.

74 Di Minno MND, Peluso R, lervolino S, et al. Weight loss and achievement of minimal disease activity in patients with psoriatic arthritis starting treatment with tumour necrosis factor $\alpha$ blockers. Ann Rheum Dis 2014;73:1157-62.

75 Messier SP, Mihalko SL, Legault C, et al. Effects of intensive diet and exercise on knee joint loads, inflammation, and clinical outcomes among overweight and obese adults with knee osteoarthritis: the idea randomized clinical trial. JAMA 2013;310:1263-73.

76 Somers TJ, Blumenthal JA, Guilak F, et al. Pain coping skills training and lifestyle behavioral weight management in patients with knee osteoarthritis: a randomized controlled study. Pain 2012;153:1199-209.

77 Riaz H, Khan MS, Siddiqi TJ, et al. Association between obesity and cardiovascular outcomes: a systematic review and meta-analysis of Mendelian randomization studies. JAMA Netw Open 2018;1:e183788-e88.

78 Abdullah A, Peeters A, de Courten M, et al. The magnitude of association between overweight and obesity and the risk of diabetes: a meta-analysis of prospective cohort studies. Diabetes Res Clin Pract 2010;89:309-19.

79 Flegal KM, Kit BK, Orpana $\mathrm{H}$, et al. Association of all-cause mortality with overweight and obesity using standard body mass index categories: a systematic review and meta-analysis. JAMA 2013;309:71-82.

80 World Health Organisation. Obesity: preventing and managing the global epidemic. who technical report series. Geneva, 2000.

81 Hart CL, Morrison DS, Batty GD, et al. Effect of body mass index and alcohol consumption on liver disease: analysis of data from two prospective cohort studies. BMJ 2010;340:c1240-c40.

82 Bagnardi V, Rota M, Botteri E, et al. Alcohol consumption and site-specific cancer risk: a comprehensive dose-response meta-analysis. Br J Cancer 2015;112:580-93.

83 Bird P, Nicholls D, Barrett R, et al. Longitudinal study of clinical prognostic factors in patients with early rheumatoid arthritis: the predict study. Int J Rheum Dis 2017;20:460-8.

84 Davis MLR, Michaud K, Sayles H, et al. Associations of alcohol use with radiographic disease progression in African Americans with recent-onset rheumatoid arthritis. $J$ Rheumatol 2013;40:1498-504.

85 Nissen MJ, Gabay C, Scherer A, et al. The effect of alcohol on radiographic progression in rheumatoid arthritis. Arthritis Rheum 2010;62:1265-72.

86 Humphreys JH, Warner A, Costello R, et al. Quantifying the hepatotoxic risk of alcohol consumption in patients with rheumatoid arthritis taking methotrexate. Ann Rheum Dis 2017;76:1509-14.

87 Doran MF, Crowson CS, Pond GR, et al. Predictors of infection in rheumatoid arthritis. Arthritis Rheum 2002;46:2294-300.

88 Lu B, Rho YH, Cui J, et al. Associations of smoking and alcohol consumption with disease activity and functional status in rheumatoid arthritis. J Rheumatol 2014;41:24-30.

89 Neogi T, Chen C, Niu J, et al. Alcohol quantity and type on risk of recurrent gout attacks: an Internet-based case-crossover study. Am J Med 2014;127:311-8.

90 Gandini S, Botteri E, lodice $\mathrm{S}$, et al. Tobacco smoking and cancer: a meta-analysis. Int J Cancer 2008;122:155-64.

91 Huxley RR, Woodward M. Cigarette smoking as a risk factor for coronary heart disease in women compared with men: a systematic review and meta-analysis of prospective cohort studies. Lancet 2011;378:1297-305.

92 Willi C, Bodenmann P, Ghali WA, et al. Active smoking and the risk of type 2 diabetes: a systematic review and meta-analysis. JAMA 2007;298:2654-64.
93 de Rooij M, van der Leeden M, Heymans MW, et al. Prognosis of pain and physical functioning in patients with knee osteoarthritis: a systematic review and metaanalysis. Arthritis Care Res 2016;68:481-92.

94 Baghdadi LR, Woodman RJ, Shanahan EM, et al. The impact of traditional cardiovascular risk factors on cardiovascular outcomes in patients with rheumatoid arthritis: a systematic review and meta-analysis. PLoS One 2015;10:e0117952.

95 Rodríguez Huerta MD, Trujillo-Martín MM, Rúa-Figueroa Î́nigo, et al. Healthy lifestyle habits for patients with systemic lupus erythematosus: a systemic review. Semin Arthritis Rheum 2016;45:463-70.

96 Villaverde-García V, Cobo-Ibáñez T, Candelas-Rodríguez G, et al. The effect of smoking on clinical and structural damage in patients with axial spondyloarthritis: a systematic literature review. Semin Arthritis Rheum 2017:46:569-83.

97 Tillett W, Jadon D, Shaddick G, et al. Smoking and delay to diagnosis are associated with poorer functional outcome in psoriatic arthritis. Ann Rheum Dis 2013;72:1358-61.

98 Agca R, Heslinga SC, Rollefstad S, et al. EULAR recommendations for cardiovascular disease risk management in patients with rheumatoid arthritis and other forms of inflammatory joint disorders: 2015/2016 update. Ann Rheum Dis 2017;76:17-28.

99 Daien $\mathrm{Cl}$, Hua C, Combe B, et al. Non-Pharmacological and pharmacological interventions in patients with early arthritis: a systematic literature review Informing the 2016 update of EULAR recommendations for the management of early arthritis. RMD Open 2017;3:e000404

100 Söderlin MK, Petersson IF, Bergman S, et al. Smoking at onset of rheumatoid arthritis $(r A)$ and its effect on disease activity and functional status: experiences from BARFOT, a long-term observational study on early RA. Scand I Rheumatol 2011;40:249-55

101 Levitsky A, Brismar K, Hafström I, et al. Obesity is a strong predictor of worse clinical outcomes and treatment responses in early rheumatoid arthritis: results from the SWEFOT trial. RMD Open 2017;3:e000458.

102 Hoving JL, Lacaille D, Urquhart DM, et al. Non-Pharmacological interventions for preventing job loss in workers with inflammatory arthritis. Cochrane Database Syst Rev 2014;11:CD010208.

103 Ramiro S, Landewé $R$, van Tubergen A, van TA, et al. Lifestyle factors may modify the effect of disease activity on radiographic progression in patients with ankylosing spondylitis: a longitudinal analysis. RMD Open 2015;1:e000153

104 Nikiphorou E, Ramiro S, Sepriano A, et al. Do smoking and socioeconomic factors influence imaging outcomes in axial spondyloarthritis? five-year data from the DESIR cohort. Arthritis Rheumatol 2020;72:1855-62.

105 Green DJ, Lewis M, Mansell G, et al. Clinical course and prognostic factors across different musculoskeletal pain sites: a secondary analysis of individual patient data from randomised clinical trials. Eur J Pain 2018:22:1057-70.

106 Chopp-Hurley JN, Brenneman EC, Wiebenga EG, et al. Randomized controlled trial investigating the role of exercise in the workplace to improve work ability, performance, and patient-reported symptoms among older workers with osteoarthritis. J Occup Environ Med 2017:59:550-6.

107 Zhou W, Chandran V, Cook R, et al. The association between occupational-related mechanical stress and radiographic damage in psoriatic arthritis. Semin Arthritis Rheum 2019;48:638-43.

108 Black C. Working for a healthier tomorrow. Norwich, 2008

109 Hiza HAB, Casavale KO, Guenther PM, et al. Diet quality of Americans differs by age, sex, race/ethnicity, income, and education level. J Acad Nutr Diet 2013;113:297-306.

110 Backholer K, Spencer E, Gearon E, et al. The association between socio-economic position and diet quality in Australian adults. Public Health Nutr 2016:19:477-85.

111 Shuval K, Li Q, Gabriel KP, et al. Income, physical activity, sedentary behavior, and the 'weekend warrior' among U.S. adults. Prev Med 2017;103:91-7.

112 O'Donoghue G, Kennedy A, Puggina A, et al. Socio-economic determinants of physical activity across the life course: A "DEterminants of Dlet and Physical ACtivity" (DEDIPAC) umbrella literature review. PLoS One 2018;13:e0190737.

113 Newton S, Braithwaite D, Akinyemiju TF. Socio-Economic status over the life course and obesity: systematic review and meta-analysis. PLoS One 2017;12:e0177151.

114 Cohen AK, Rai M, Rehkopf DH, et al. Educational attainment and obesity: a systematic review. Obes Rev 2013;14:989-1005.

115 Hiscock R, Bauld L, Amos A, et al. Socioeconomic status and smoking: a review. Ann N Y Acad Sci 2012;1248:107-23.

116 Collins SE. Associations between socioeconomic factors and alcohol outcomes. Alcohol Res 2016:38:83-94.

117 Warren JR, Hoonakker P, Carayon P, et al. Job characteristics as mediators in SES-health relationships. Soc Sci Med 2004;59:10.1016/j.socscim ed.2004.01.035:1367-78.

118 Gwinnutt JM, Verstappen SM, Humphreys JH. The impact of lifestyle behaviours, physical activity and smoking on morbidity and mortality in patients with rheumatoid arthritis. Best Pract Res Clin Rheumatol 2020;34:101562.

119 Knittle K, De Gucht V, Maes S. Lifestyle- and behaviour-change interventions in musculoskeletal conditions. Best Pract Res Clin Rheumatol 2012;26:293-304. 